\title{
Fluid-structure interaction mechanisms for close-in explosions
}

\author{
Andrew B. Wardlaw, Jr. and J. Alan Luton \\ Naval Surface Warfare Center, Indian Head Division, \\ Warhead Dynamics Division, Code 420, 101 Strauss \\ Avenue, Indian Head MD 20640-5035, USA \\ Tel.: +13017442286
}

Received 24 January 2000

Revised 22 May 2000

This paper examines fluid-structure interaction for close-in internal and external underwater explosions. The resulting flow field is impacted by the interaction between the reflected explosion shock and the explosion bubble. This shock reflects off the bubble as an expansion that reduces the pressure level between the bubble and the target, inducing cavitation and its subsequent collapse that reloads the target. Computational examples of several close-in interaction cases are presented to document the occurrence of these mechanisms. By comparing deformable and rigid body simulations, it is shown that cavitation collapse can occur solely from the shock-bubble interaction without the benefit of target deformation. Addition of a deforming target lowers the flow field pressure, facilitates cavitation and cavitation collapse, as well as reducing the impulse of the initial shock loading.

\section{Introduction}

Accurate prediction of underwater explosion damage against surface ships and submarines is an important aspect of naval weapon design. The stiffness of water makes this a difficult problem; target deformation modifies the pressure in the surrounding fluid, changing the explosive loading. Thus, it is necessary to couple the fluid/explosive modeling with the structure simulation.

Close-in explosions add the complication of the shock-bubble interaction to fluid-structure interaction. This is unlike distant explosions, where fluid-structure interaction is a consequence of target deformation. In the close-in case, the primary shock reflects off the target and interacts with the explosion bubble, creating an expansion that travels towards the target, reducing the pressure between the bubble and target. This ex- pansion reflects off the target as an expansion, driving surface pressures lower. A consequence of the formation of low-pressure regions in the flow field is the advent of cavitation zones that, when surrounded by highpressure fluid, collapse to generate a secondary shock that can reload the target. The bubble-shock interaction mechanism is operative even with a rigid target. Target flexibility augments the pressure reduction by inducing wall motion and ultimately expanding the cavitation zone.

The objective of this paper is to demonstrate the influence of the shock-bubble interaction and the subsequent cavitation collapse on fluid-structure interaction. This is accomplished by applying the coupled GEMINI-DYNA_N hydrocode to a series of test cases: an internal explosion within a single wall cylinder, an internal explosion inside a double wall cylinder and an external explosion near a flat plate. By comparing the single wall cylinder results to experiment, the qualitative validity of shock-bubble interaction predictions and the cavitation model are established. The additional examples highlight the impact of these phenomena on fluid-structure interaction. To distinguish between the influence of the shock-bubble interaction and target deformation, the single wall cylinder and flat plate cases are also simulated using rigid structures.

\section{Methodology}

The GEMINI-DYNA $N$ coupled code describes the fluid using the GEMINI Euler Equation solver and the target using the DYNA_N finite element code. These codes are run as separate, parallel processes with information exchanged at the end of every fluid computational step; DYNA_N is allowed to sub-cycle if necessary. Currently the data exchange is accomplished via files but use of the Message Passing Interface (MPI) system is planned for the future. The protocol that couples these processes is termed the Standard Coupler Interface: node forces are passed from the fluid to the Lagrange code and node velocities and locations 
are returned. The implementation of Standard Coupler Interface in DYNA_N requires the addition of subroutines that export the node locations and velocities and import the node loads; complimentary subroutines exist in GEMINI. The intersection of the structure with the fluid grid is accomplished exclusively by routines in GEMINI. This minimizes the amount of data exchanged and allows the Standard Coupler Interface to be independent of the fluid grid type.

The GEMINI Euler solver is a time split, higher order Godunov method based on the Collela [1] MUSCL scheme. This method has been modified to handle mixed cells with the Lagrange plus re-map procedure described in [2]. The GEMINI Euler solver operates on a fixed, Cartesian product grid (rectangular cells in 2D), which facilitates the treatment of large scale structural deformation and rupture without the need to re-grid. However, the structure is not aligned with the mesh and partially blocked cells arise, which must be treated in a special manner. The current approach is to construct a reflected flow field using the cells inside the structure to store the reflected fluid state.

DYNA_N [3,4], the Navy version of the DYNA code, is an explicit finite element code for problems where high rate dynamic or stress wave propagation effects are important. The available element formulations include one-dimensional truss and beam elements, twodimensional quadrilateral and triangular shell elements, and three-dimensional continuum elements. Many material models are available to represent a wide range of material behavior including elasticity, plasticity, composites, thermal effects, and rate dependence. In addition, DYNA_N has a sophisticated contact interface capability, including frictional sliding and single surface contact. This handles arbitrary mechanical interactions between independent bodies or between two portions of one body.

A modified form of the Tillotson equation of state [5] is used to model water:

$$
\begin{aligned}
p= & \max \left(p_{0}+\omega \rho\left(e-e_{0}\right)+A \mu+B \mu^{2}\right. \\
& \left.+C \mu^{3}, p_{\text {cav }}\right) ; \\
\mu= & \rho / \rho_{0}-1
\end{aligned}
$$

where $p$ is pressure, $\rho$ is density, $e$ is energy and the remaining terms are constants. In cgs units their values are: $p_{0}=1\left(10^{6}\right), \omega=0.28, e_{0}=3.542\left(10^{9}\right)$, $A=2.2\left(10^{10}\right), B=9.94\left(10^{10}\right), C=1.457\left(10^{11}\right)$ and $p_{c a v}=5\left(10^{4}\right)$. The first term in the $\max ()$ function is the Tillotson equation of state for water while the second enforces a cavitation lower pressure limit.
The assigned value of $p_{\text {cav }}$ corresponds to 0.05 bars. However, it has little impact on the solution as long as it is selected to be positive and much less than 1 bar.

Bulk cavitation regions are viewed as consisting of bubbly water (e.g., see Cole [6]), rather than a gaseous or void region. Here rapid volume expansion with a small change in pressure is permitted via growth in the number and size of the bubbles without placing the water in tension. The bulk cavitation model defined in Eq. 1 by the lower pressure limit of $p_{\text {cav }}$ fulfills the basic requirement for cavitation simulation: it allows water at low pressure to significantly expand without going into tension. However, it introduces other characteristics that are not realistic. In particular, prescription of a uniform pressure throughout a variable density cavitation region implies a sound speed of zero and changes the form of the equations from hyperbolic to parabolic. In order to be compatible with most numerical methods, a positive sound speed is required everywhere throughout the flow field. The present approach is to compute sound speed via $\partial p /\left.\partial \rho\right|_{s}$, without consideration of the cavitation limit.

\section{Fluid-structure interaction mechanisms}

The traditional view of fluid-structure interaction is depicted in Fig. 1. The explosion shock loads the target and deforms it, creating an extra volume to be filled by water. Water immediately fills this region, reducing the local density and creating a low pressure zone next to the target that may be cavitated. This unloads the target causing it to rebound and to collapse the low pressure, possibly cavitated region, thus reloading the target.

A close-in explosion flow field contains features not accounted for in the traditional view of fluid-structure interaction. After the explosion shock reflects off the target, it interacts with the explosion bubble. At this time, the density in the bubble is much less than that of the surrounding water and the bubble interface behaves approximately as a free surface with regard to the water shock. Thus, the shock is reflected as an expansion traveling back towards the target with only a weak shock transmitted into the bubble. This expansion reduces the pressure between the bubble and target, creating a zone susceptible to cavitation. A cavitation zone that forms in this region is surrounded by much higher pressure fluid and is likely to collapse, generating a spherical shock that reloads the adjacent target.

Fluid-structure interaction with a rigid target is illustrated in Fig. 2. This figure depicts the expansion gen- 

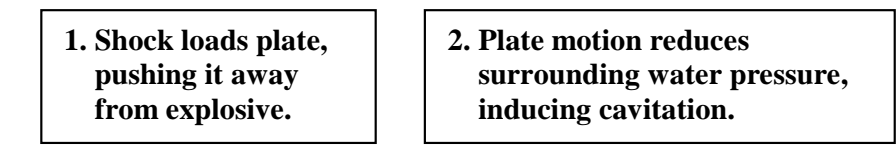
3. Plate rebounds, eliminating cavitation region and reloading.
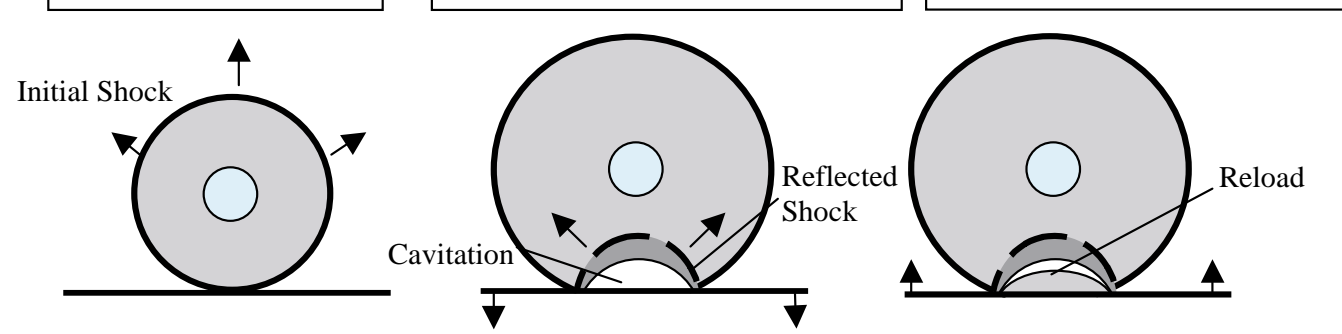

Fig. 1. Traditional view of fluid-structure interaction.

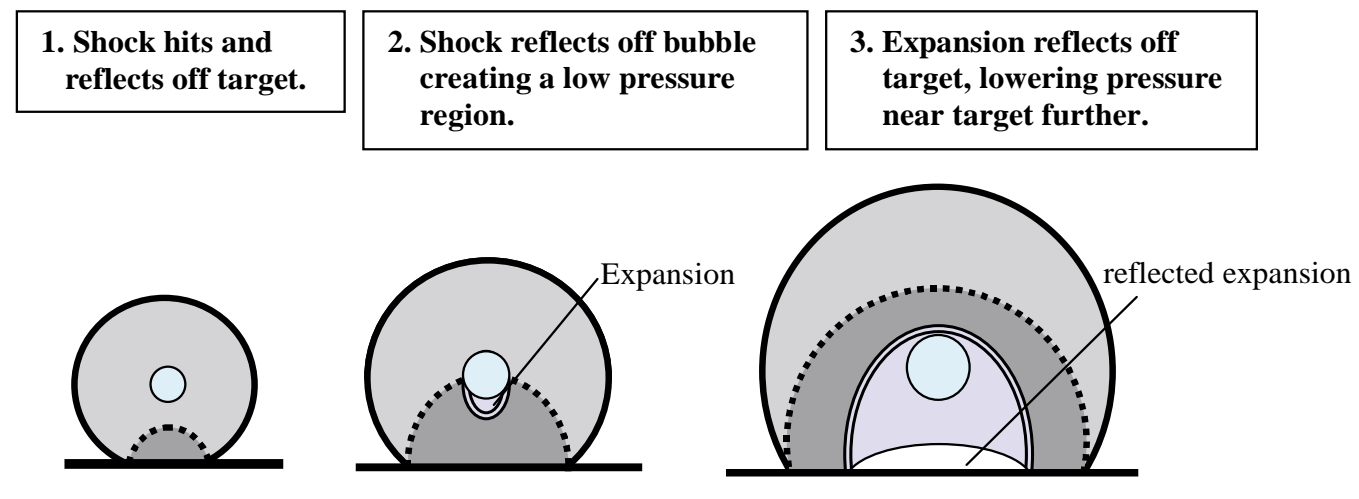

Fig. 2. Close-in fluid-structure interaction for a rigid target.
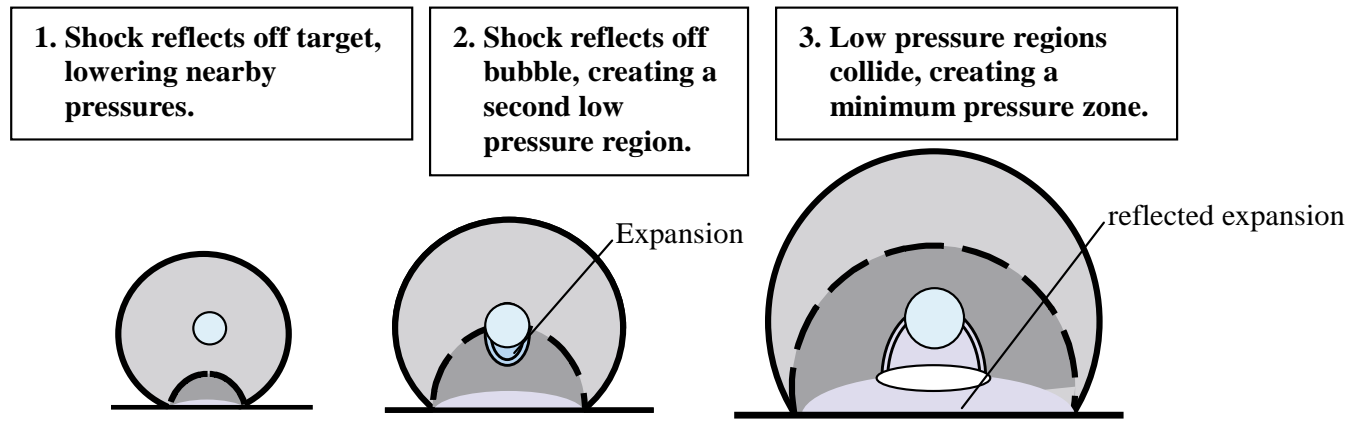

Fig. 3. Close-in fluid-structure interaction for a deforming target.

erated by the shock-bubble interaction as well as the subsequent reflection of this expansion off the target, which creates an even lower pressure zone on the target surface. This low pressure zone is subject to cavitation and subsequent collapse that reloads the target, even without target deformation.

Replacement of the rigid target with a deformable one changes the fluid-structure interaction as is shown in Fig. 3. The initial shock impact deforms the target, creating a low pressure region on the target surface while the shock-bubble interaction creates an additional low pressure region. These regions move towards one another, interacting somewhere between the bubble and the target. This creates a new lower pressure zone susceptible to cavitation.

The scenario shown in Fig. 3 presumes a relatively stiff target. As the flexibility of the target increases, plate motion following the initial shock motion will increase as will the reduction in pressure near the target. In the limit of a target that exhibits no resistance to 


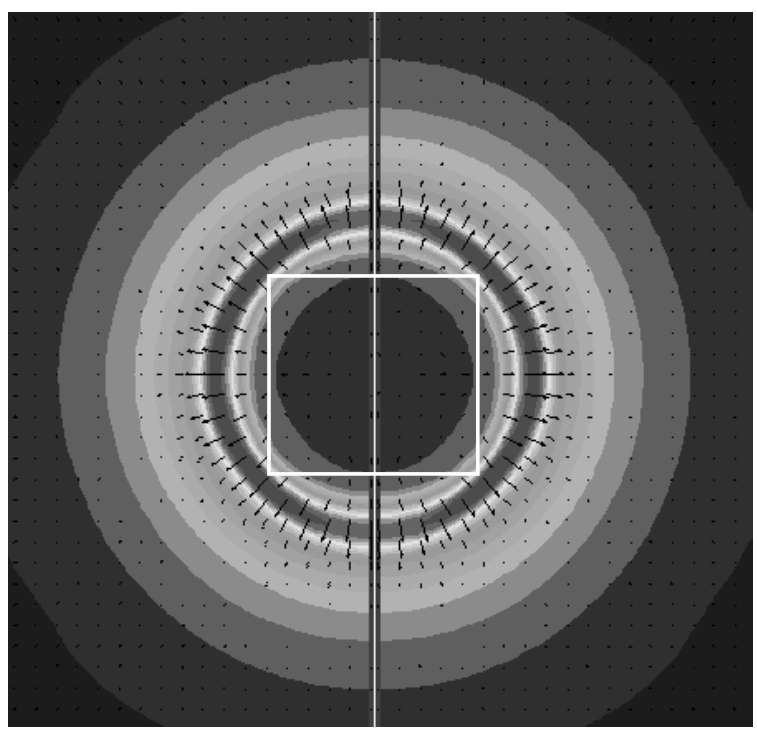

Fig. 4. Cavitation Collapse Shock.

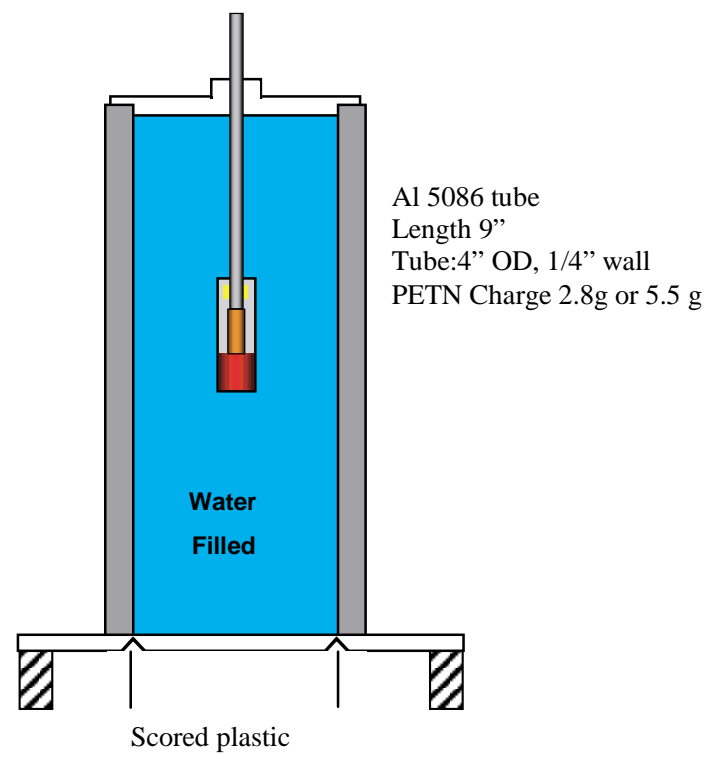

Fig. 5. Single Wall Cylinder.

deformation, the fluid will behave as though the target is a free surface and the initial shock will be reflected as an expansion.

Cavitation collapse occurs when high-pressure fluid, surrounding a cavitated zone, flows into this zone. This fluid converges at a central point within the zone generating an outward moving spherical shock that can reload a nearby target. The strength of the shock is determined by the size of the cavitated zone as well as the difference in pressure levels between the zone and the surrounding water. Collapse of a cylindrical cavitation zone surrounded by stationary water at a pressure of 100 bars is demonstrated in Fig. 4. Here the color contours represent pressure levels and the arrows the velocity vectors. The initial cavitated area, with a density of $0.8 \mathrm{~g} / \mathrm{cm}^{3}$, is contained within the cylinder outlined in white. Note that a cavitation-like collapse can occur in the absence of a cavitation model or cavitation; it simply requires a low pressure region to be surrounded by a high pressure one. Collapse occurs when the fluid rushes in to fill the low pressure region.

\section{Single walled cylinder}

The experimental arrangement is illustrated in Fig. 5 and consists of a water filled aluminum cylinder with a $4^{\prime \prime}$ outer diameter and a $0.25^{\prime \prime}$ wall thickness. This cylinder contains approximately $3.0 \mathrm{~g}$ of PETN explosive plus detonator, located at the cylinder center, along the midline. The deformation history of the cylinder outer wall and surface pressure near the inner wall are measured following the charge detonation.

This test, described in [7,8], provides wall velocity and pressure histories that can be used to verify the cavitation collapse and reloading phenomena. Detailed comparison of calculation and experiment have been previously reported in [9-11]. In this paper the emphasis is on relating measurements to the fluid structure mechanisms shown in Figs 2 and 3. A rigid wall cylinder case is also presented to differentiate between shock-bubble interaction and wall motion effects. The computation domain includes an outer cylinder of air at whose outer boundaries flow field extrapolation boundary conditions are applied.

The flow field pressure contours within the cylinder are displayed in Fig. 6 at $30 \mu \mathrm{s}, 40 \mu \mathrm{s}, 60 \mu \mathrm{s}, 80 \mu \mathrm{s}$ and $90 \mu \mathrm{s}$. The white regions in this figure are cavitated zones and the air region surrounding the cylinder has been truncated. The flow at $30 \mu$ s shows the reflected shock from the cylinder as it intersects the bubble for the first time. A cavitation region has formed at the wall because of wall motion and a second cavitation region is starting to form behind the reflected expansion. These regions merge at $40 \mu$ s to form a large zone, surrounded by high pressure fluid, that collapses at $90 \mu \mathrm{s}$, generating a collapse shock that reloads the cylinder.

The calculated and measured midline outer wall velocity and inner wall pressure histories are shown in Fig. 7. The measured and calculated surface pressures 
$30 \mu \mathrm{s}$

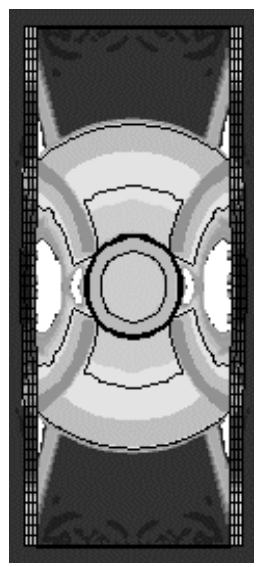

$40 \mu \mathrm{s}$

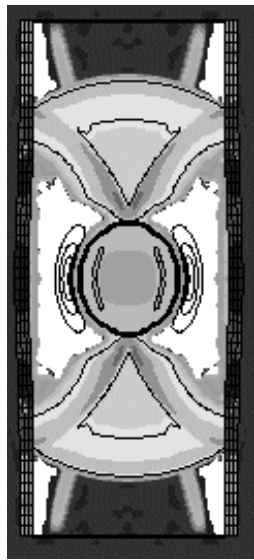

$60 \mu \mathrm{s}$

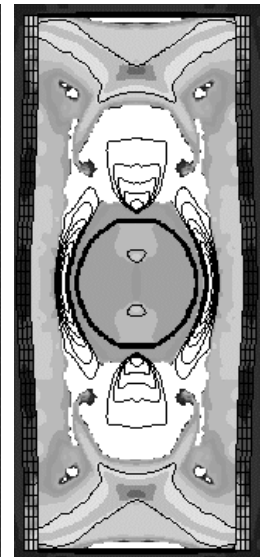

$80 \mu \mathrm{s}$

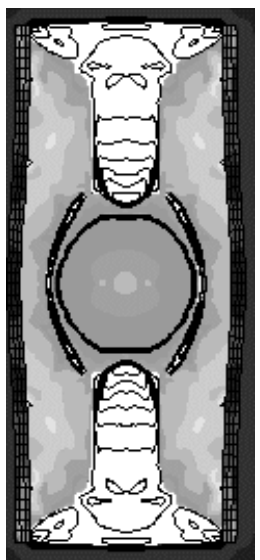

$90 \mu \mathrm{s}$

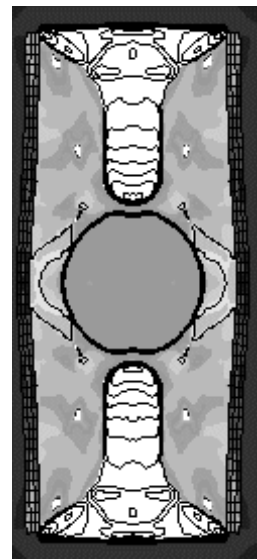

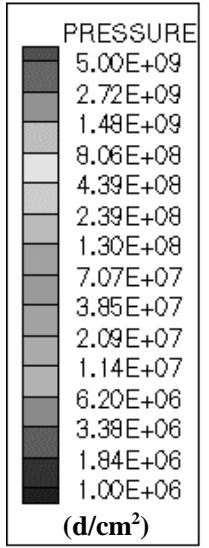

Fig. 6. Pressure contours in the evolving flow field of the single wall cylinder flow case.

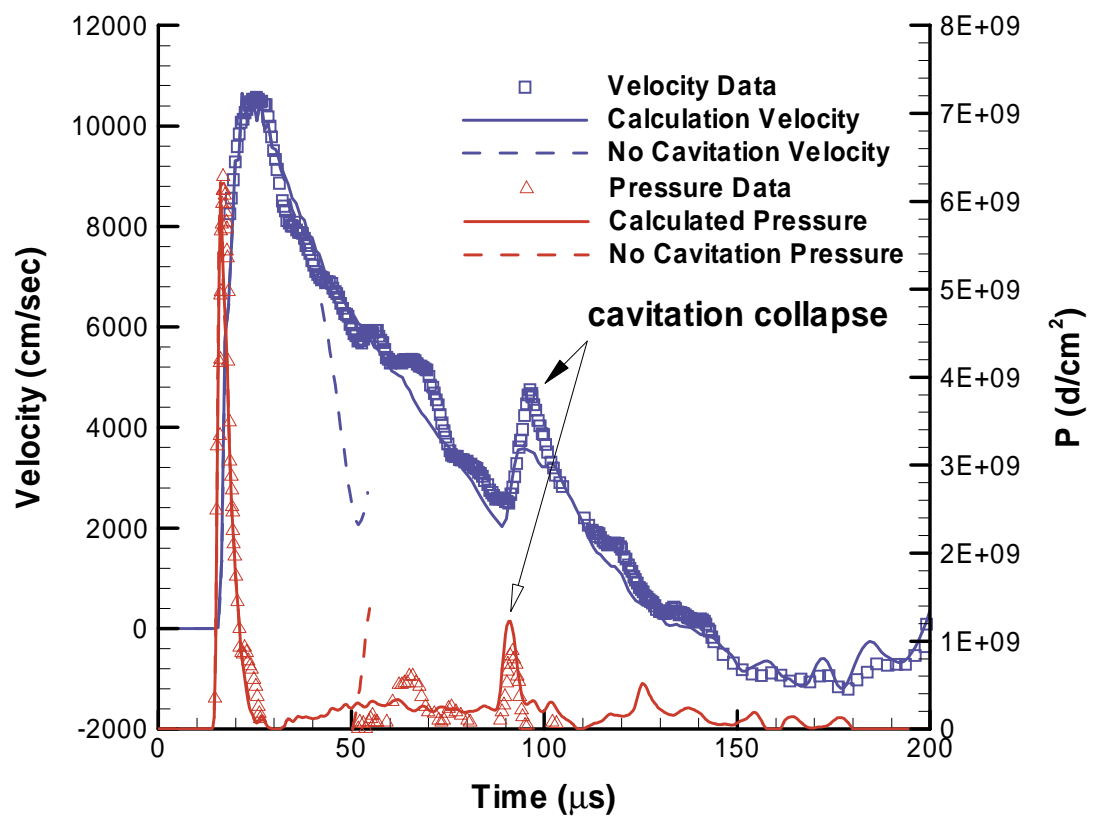

Fig. 7. Single wall cylinder pressure and velocity history.

exhibit a pressure pulse at about $90 \mu \mathrm{s}$, which is coincident with the predicted cavitation collapse event. This supports the conclusion that the observed change in wall velocity and surface pressure at this time is a consequence of cavitation collapse. Furthermore, it lends credence to the proposition that coupled hydrocodes, using a simple cavitation model, are capable of capturing such a phenomenon. However, a smaller measured pressure rise and outward wall velocity at $60-70 \mu \mathrm{s}$ is not reflected in the calculations.

A calculation without a cavitation model is shown in
Fig. 7 for the single walled cylinder. A consequence of this omission is that water experiences tension, rather than cavitation. Although this simulation terminates at $65 \mu \mathrm{s}$, the solution at this time demonstrates cylinder reloading. Here a low pressure region between the bubble and cylinder wall is annihilated by the inflow of surrounding high pressure fluid. The impact of the omission of the cavitation model is to limit the water expansion, which curtails cylinder deformation. This accounts for the decrease in the wall velocity for $t>$ $50 \mu$ s shown in Fig. 7 . 


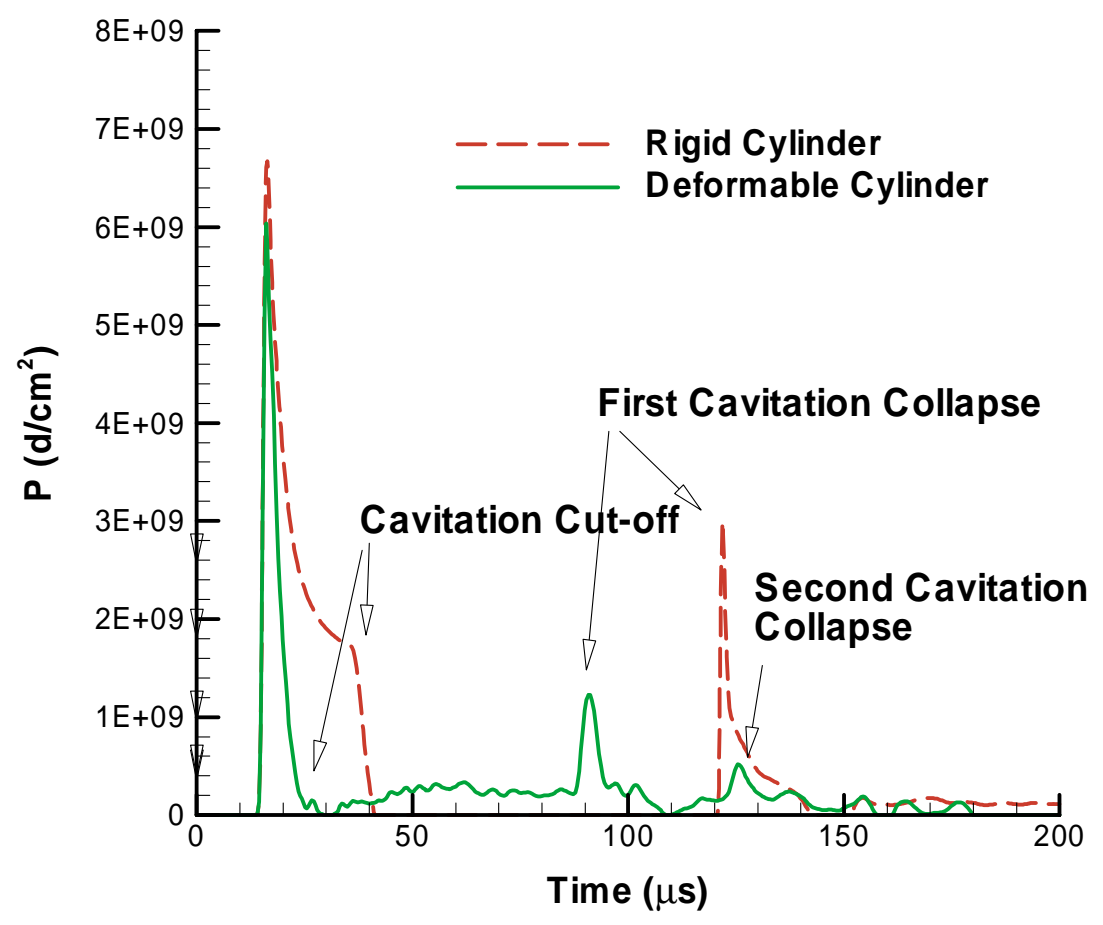

Fig. 8. Inner wall pressure histories for the deformable and rigid cylinder cases.

$30 \mu \mathrm{s}$

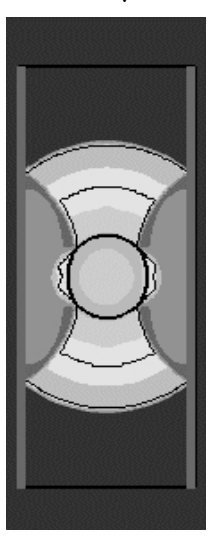

$60 \mu \mathrm{s}$

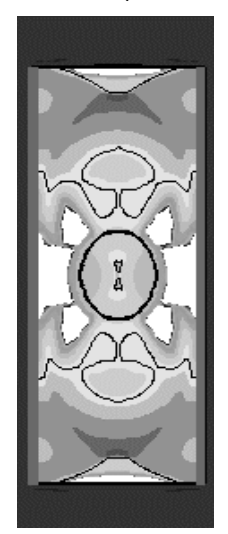

$90 \mu \mathrm{s}$

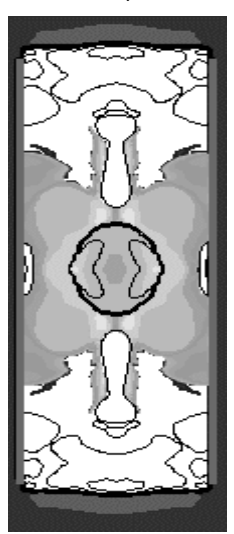

$130 \mu \mathrm{s}$

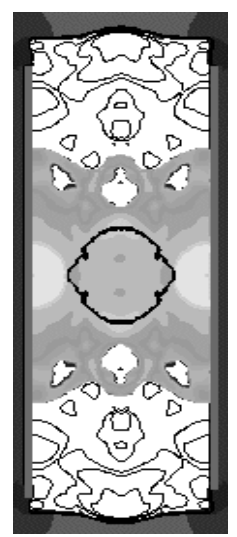

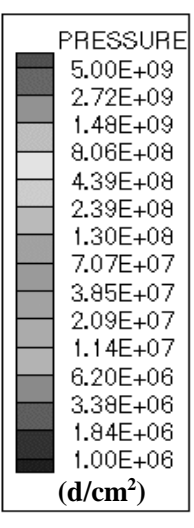

Fig. 9. Cavitation collapse in the rigid, single wall cylinder case.

Rigid, single cylinder results are shown in Figs 8 and 9. The former illustrates the centerline wall pressure history for the rigid and deforming cases, while the later presents the rigid wall cylinder flow field at $30 \mu \mathrm{s}$, $60 \mu \mathrm{s}, 90 \mu \mathrm{s}$ and $130 \mu \mathrm{s}$. The occurrence of cavitation collapse in the rigid cylinder case is clearly visible in Fig. 9. At $30 \mu \mathrm{s}$, the reflected shock can be seen interacting with the bubble, a process that does not lower the pressure sufficiently to induce cavitation. Instead, cavitation occurs after the expansion reflects off the cylinder wall. The resulting cavitation region grows, as is shown at $60 \mu \mathrm{s}$, and collapses near $120 \mu \mathrm{s}$. The wall pressure, shown in Fig. 8, reflects the formation of the cavitation region and the first cavitation collapse.

The deforming wall midline pressure history is compared to the rigid one in Fig. 8. Note that the initial shock pressure peak at the wall is similar in both cases. However, the deforming case decays more quickly in response to wall motion. By contrast, the rigid case retains a high level until the advent of cavitation cut- 

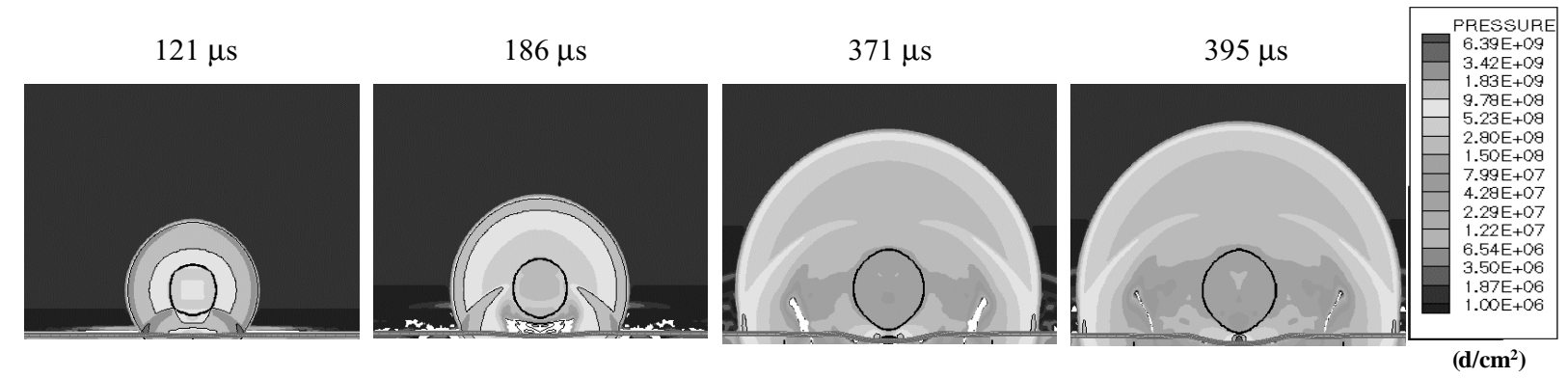

Fig. 10. Flat plate cavitation formation and collapse.

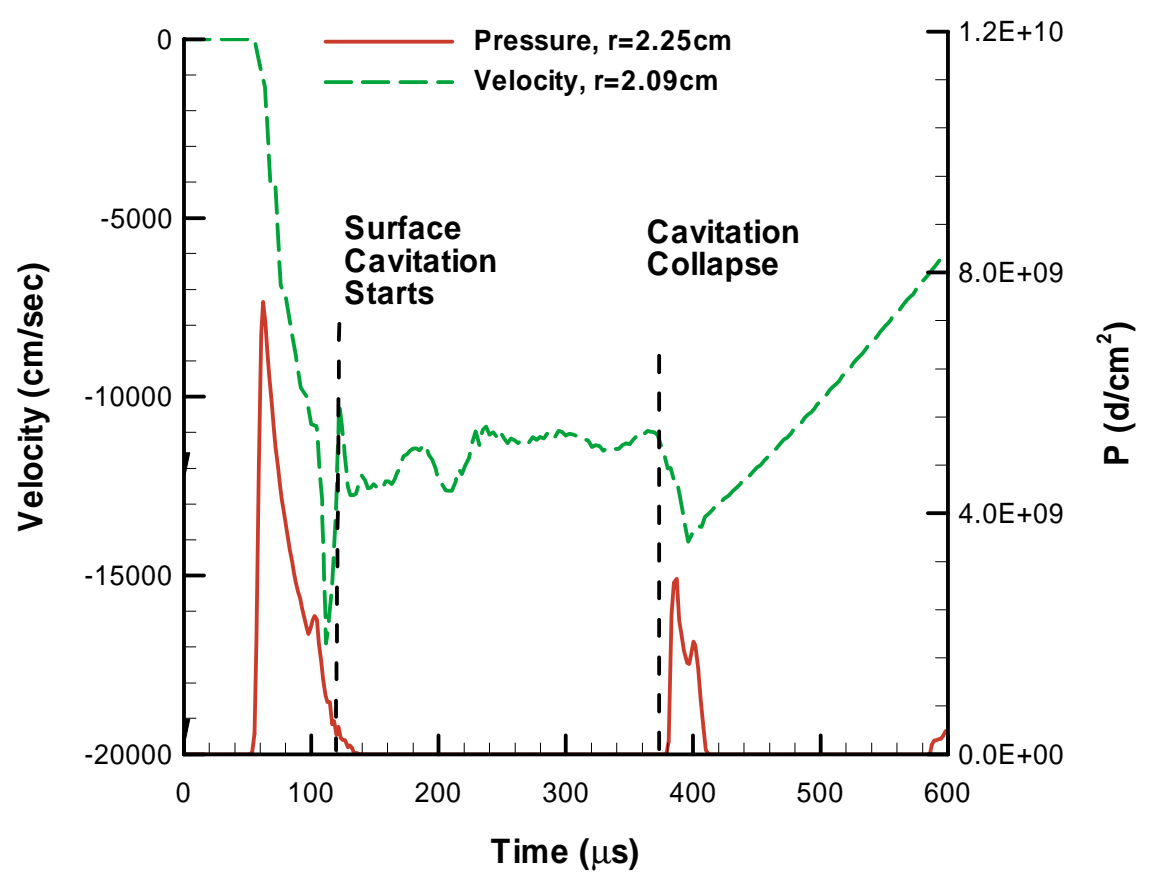

Fig. 11. Flat plate centerline velocity and pressure history.

off. The peak cavitation collapse pressure, shown in Fig. 8, is higher in the rigid body case. Here, the collapsing cavitation zone is adjacent to the wall, while in the deformable case it is midway between the bubble and cylinder.

\section{Flat plate}

The previous example demonstrates cavitation collapse and reloading for an internal explosion. The flat plate case illustrates that it also occurs in an external explosion, which is of greater interest in weapon design. There are no supporting measurements for this case and credence in the computations rests on the success achieved in the previous, single wall cylinder case.
This calculation places a $737 \mathrm{~g}$ charge of TNT at $24.75 \mathrm{~cm}$ above a circular steel plate, $55 \mathrm{~cm}$ in diameter and $2 \mathrm{~cm}$ in thickness. Results from this run are shown in Figs 10 and 11. The former figure depicts the flow field at $121 \mu \mathrm{s}, 186 \mu \mathrm{s}, 371 \mu \mathrm{s}$ and $395 \mu \mathrm{s}$, while the latter displays plate centerline velocity and pressure history. Fig. 10 indicates that cavitation first occurs between the bubble and plate. This follows the merger of the shock-bubble interaction expansion and the low pressure region generated by the plate deformation, visible at $121 \mu \mathrm{s}$. This cavitation zone grows to peak size at about $180 \mu$ s and eventually collapses at about $360 \mu \mathrm{s}$. The collapse first occurs at the outer edges of the cavitation region and then progresses inwards, forming a high-pressure torus under the bubble that contracts to the centerline at $395 \mu \mathrm{s}$. The shock 


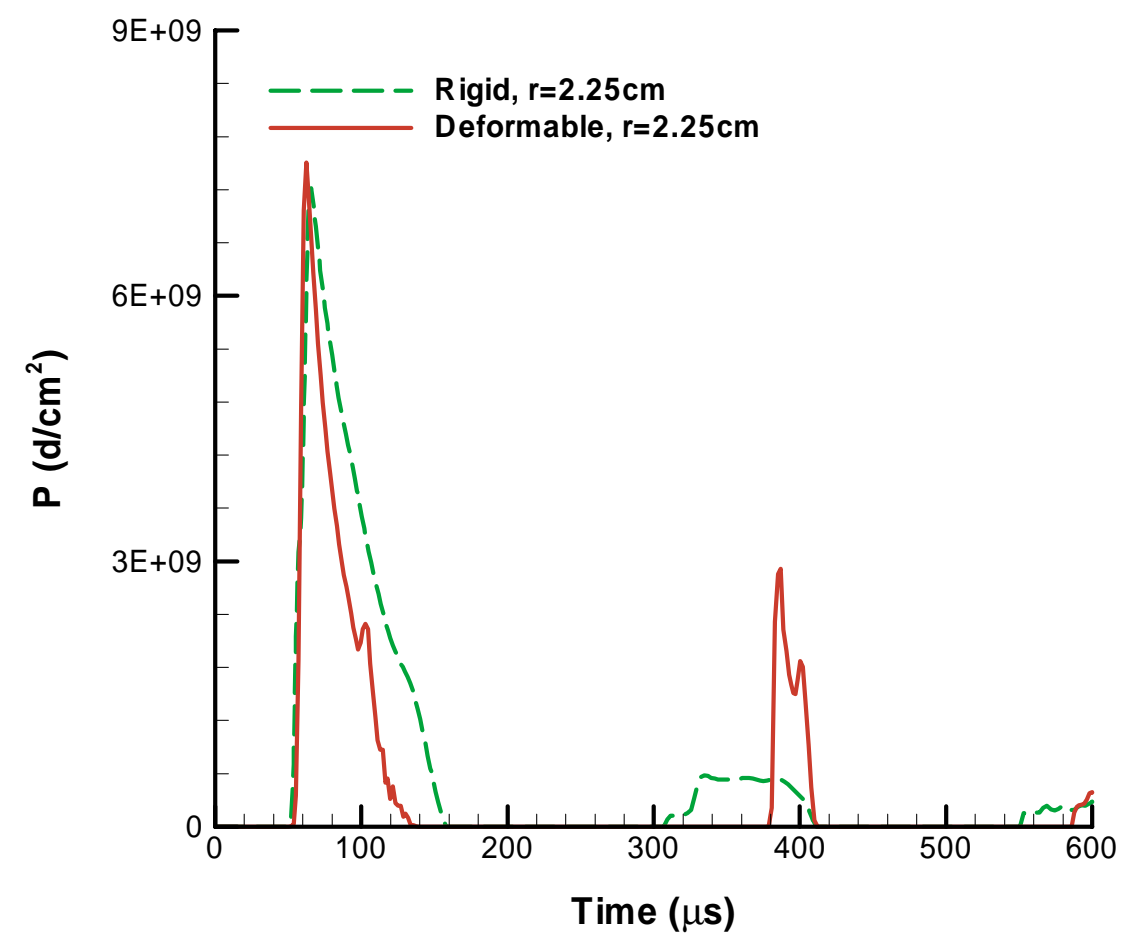

Fig. 12. Centerline wall pressure histories for the rigid and flexible plates.
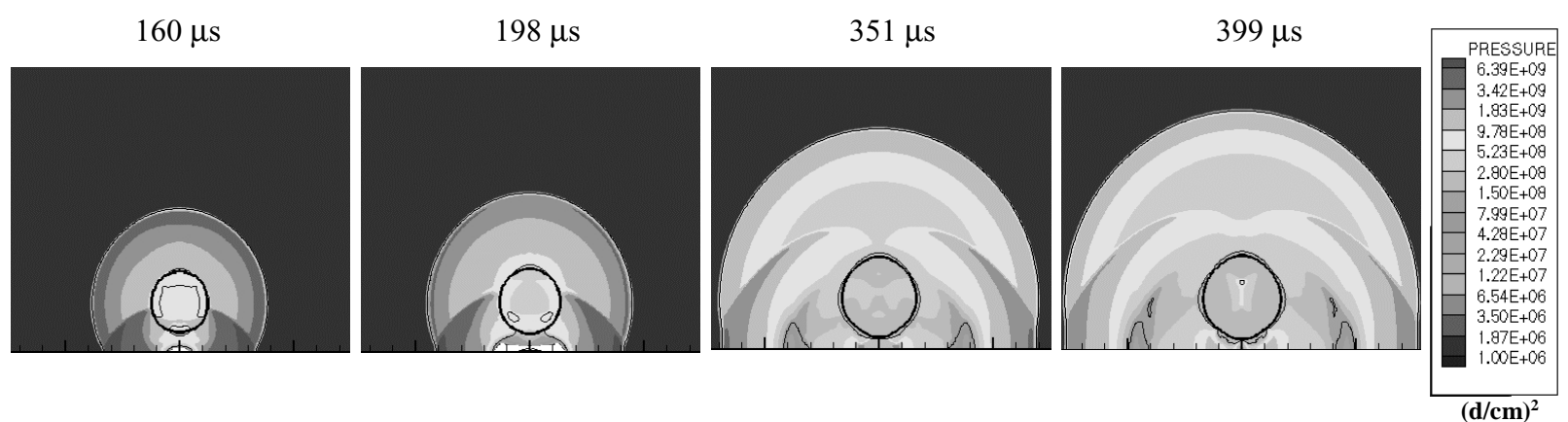

Fig. 13. Rigid flat plate cavitation formation and collapse.

formed by this collapse interacts with the explosion bubble again, producing a second cavitation region that collapses near $600 \mu$ s (not shown). Fig. 11 demonstrates that the pressure rise generated by the first collapse alters the plate velocity. Here pressure and velocity are shown near the center of the plate and the symbol $r$ is the distance from the center of the plate. The collapse pressure is highest at the centerline and diminishes with increasing $r$.

Rigid, flat plate predictions are shown in Figs 12 and 13. The latter figure outlines the flow field evolution and demonstrates cavitation collapse at about $350 \mu \mathrm{s}$. In the rigid calculation, cavitation occurs adjacent to the plate as a consequence of the reflection of the expansion off the plate. This is in contrast to the deforming plate, where cavitation forms between the bubble and plate from the merger of the shock-bubble interaction expansion and the expansion generated by plate motion. The ensuing cavitated region formation and collapse occur at similar times in the deforming and rigid plate cases. Fig. 12 demonstrates that the rigid and deforming plate initial shock pressure peaks are of similar magnitude; however, the deforming plate values decrease more rapidly as a consequence of plate motion. 


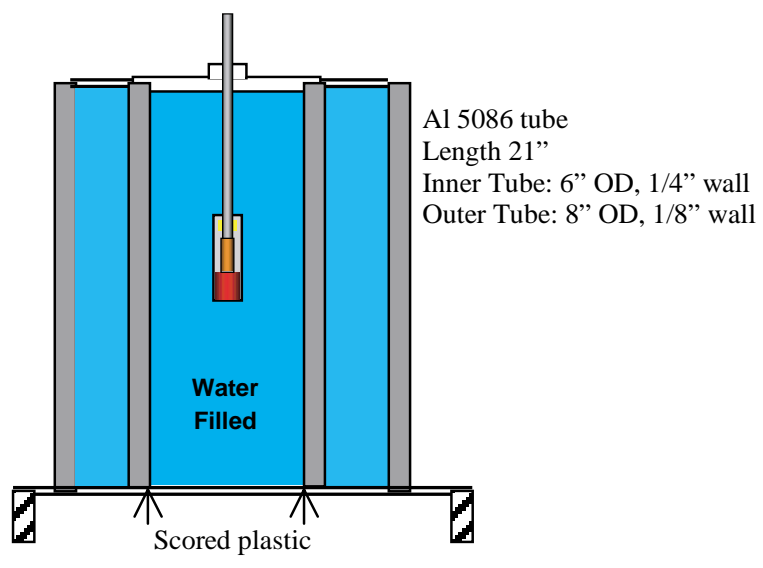

Fig. 14. Double Walled Cylinder.

\section{Double walled cylinder}

The doubled wall cylinder, shown in Fig. 14, is similar to the single wall cylinder with the exception that an additional outer cylinder has been added. The outer cylinder outer diameter is $8^{\prime \prime}$, with wall thickness of $1 / 8^{\prime \prime}$, while the inner cylinder outer diameter is $6^{\prime \prime}$ with a wall thickness of $1 / 4^{\prime \prime}$. Both tubes are water-filled with a $12 \mathrm{~g}$ charge of PETN plus detonator is placed at the center. The computational domain includes a cylinder of air that surrounds the test device.

The double walled cylinder is the internal analog of an explosion near a double hulled vessel. Although this arrangement has been tested [12], velocity data useful for corroborating cavitation collapse and reloading is not available. Comparison of prediction and calculations for other quantities is given in $[10,11]$. The present paper focuses on an analysis of the computed flow field, shown in Fig. 15.

Figure 15 depicts the flow field evolution at $45 \mu \mathrm{s}$, $55 \mu \mathrm{s}, 80 \mu \mathrm{s}, 105 \mu \mathrm{s}$, and $120 \mu \mathrm{s}$ while the wall velocity and surface pressures on the inner cylinder are displayed in Figs 16 and 17. The interaction of the reflected shock with the bubble occurs at $45 \mu \mathrm{s}$. At this time, a cavitation region has formed behind the shock and the component of the shock transmitted through the inner wall has just reached the outer wall. At $55 \mu \mathrm{s}$, the shock transmitted into the outer cylinder has reflected off the outer wall and returned to the inner wall, accelerating it inwards. This generates a second shock, inside the inner cylinder, that moves inwards and reflects off the initial cavitation zone that formed at $45 \mu \mathrm{s}$. At $80 \mu$ s a new outer cavitation zone is visible, spawned by this interaction. The initial cavitation zone collapses at $105 \mu \mathrm{s}$, causing the new zone to collapse shortly after and then to reload the inner cylinder at $110 \mu \mathrm{s}$.
The computed pressure and velocity histories on the inner wall of the double walled cylinder, along the midline, are shown in Figs 16 and 17. The dip in the inner wall velocity that occurs at $60 \mu$ s is a consequence of the arrival of the reflected outer cylinder shock visible in Fig. 15 at $55 \mu$ s. Cavitation collapse, visible at $120 \mu \mathrm{s}$ in this figure, increases the pressure on the inside of the wall and accelerates it outwards.

\section{Summary and conclusions}

Fluid-structure interaction for close-in explosions has been studied using the GEMINI-DYNA $\mathrm{N}$ coupled hydrocode. Here water is modeled using a modified Tillotson equation of state. Bulk cavitation occurs in explosion flow fields and these regions are simulated with a pressure floor cavitation model. Bulk cavitation regions are thought to consist of bubbly water, in which the low density, compressible bubbles allow the water to expand. There are some theoretical issues with this model; however, it fulfills the basic requirement of allowing water to expand at low pressures without inducing tension.

Coupled hydrocode calculations have been completed for explosions inside a single and double wall cylinder, as well as near a flat plate. Results of the numerical simulations indicate that the fluid-structure interaction of a close-in explosion is dominated by the shock-bubble interaction. This interaction occurs after the initial shock has been reflected off the target and has arrived back at the explosion bubble. The subsequent shock-bubble interaction generates an expansion, directed back towards the target, that leaves a lowpressure region between the bubble and target. This region often cavitates, followed by cavitation collapse that can reload the structure. The collapse occurs when the high-pressure fluid surrounding the cavitated zone rushes in to fill it. This generates an outward propagating spherical shock. Comparison of the single wall cylinder calculations to experiment confirms the occurrence of cavitation reloading and validates the ability to capture such phenomenon using a pressure floor cavitation model.

Rigid body simulations of the single cylinder and flat plate cases indicate that the shock-bubble interaction alone is sufficient to unload the target. Surface pressure traces demonstrate that the initial shock load is curtailed by the formation of the cavitation region caused by the shock-bubble interaction. Reloading occurs as a 


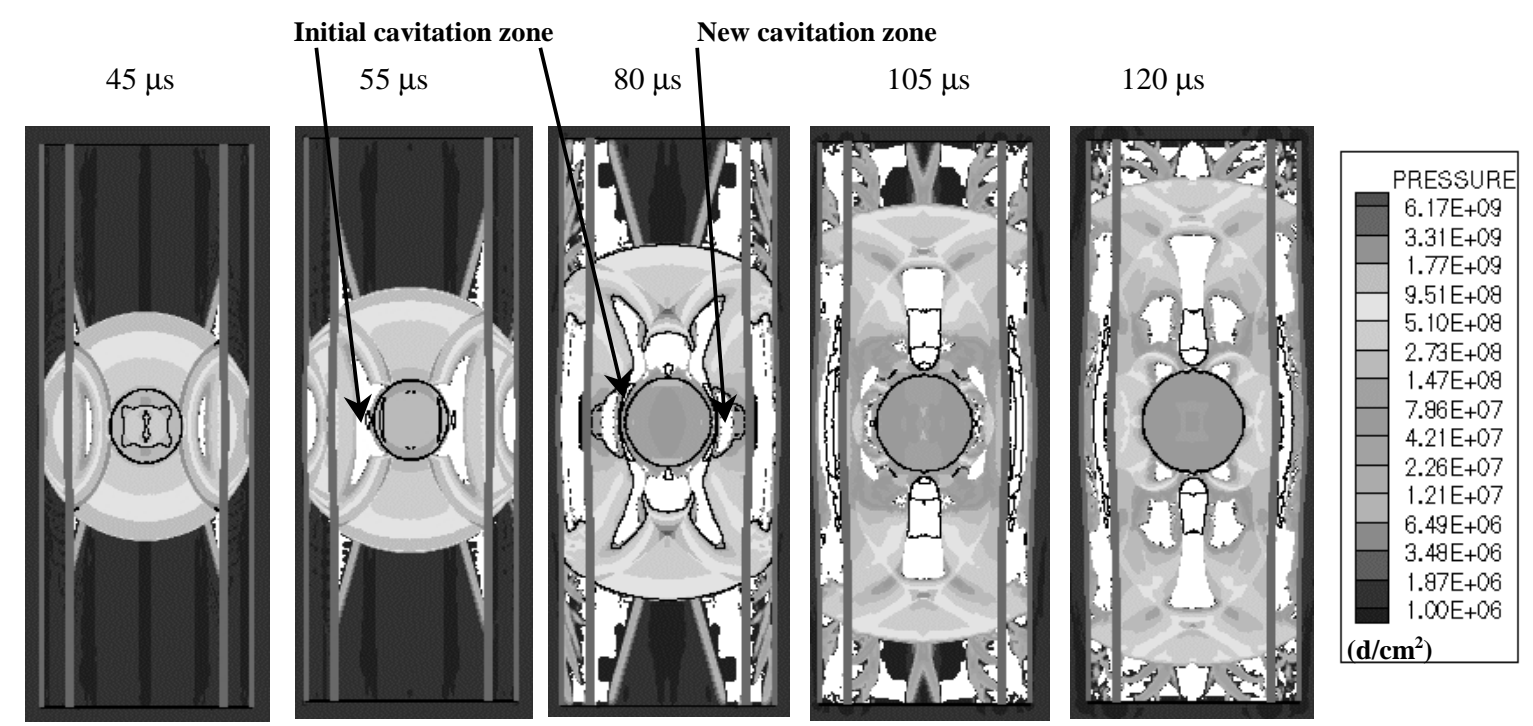

Fig. 15. Pressure contours illustrating the double cylinder flow field cavitation collapse.

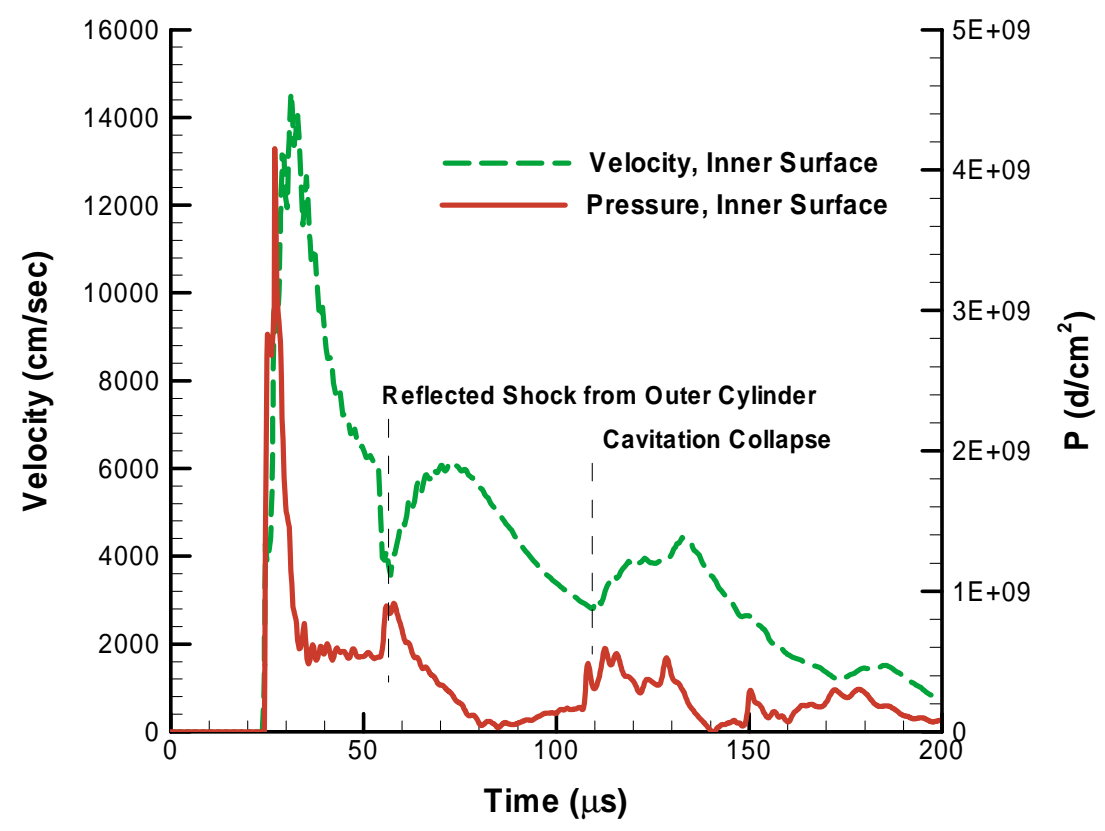

Fig. 16. Computed velocity of the inside of the inner wall at the midline for the double wall cylinder.

consequence of cavitation collapse, facilitated by the high pressures surrounding the cavitation region.

Introduction of a deformable target diminishes the length of the initial shock loading, although it does not lower the initial peak pressure. Additionally, target deformation increases the local fluid volume and lowers pressures near the explosion and target. This facilitates the formation of cavitation regions with the potential to collapse and reload the target. The initial shock loading is the principle mechanism for deforming the target; however, reloading can impart a significant change in the target surface velocity.

\section{Acknowledgement}

This work was sponsored by Judah Goldwasser, of the Office of Naval Research, Code 333. 


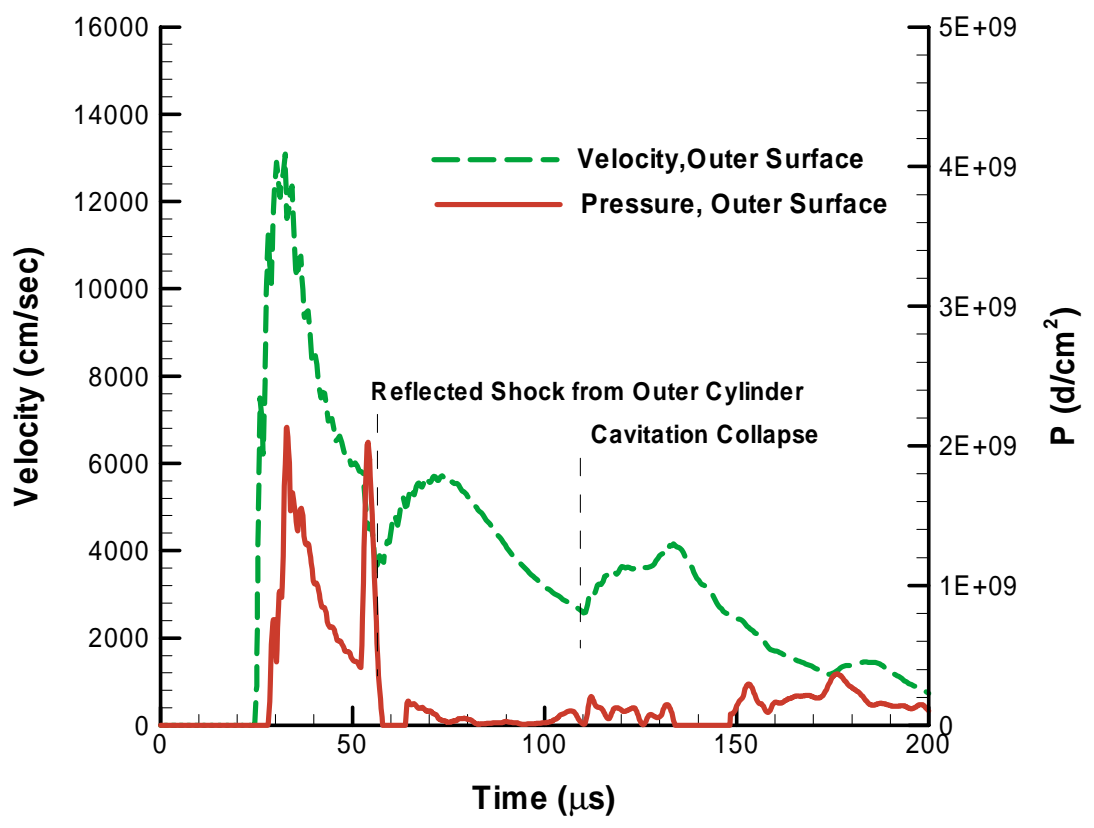

Fig. 17. Computed velocity of the outside of the inner wall at the midline for the double wall cylinder.

\section{References}

[1] P.A. Collela, Direct Eulerian MUSCL Scheme for Gas Dynamics, SIAM J. STAT COMPUT 6 (Jan. 1985), 1

[2] A. Wardlaw, The 1-D Godunov Hydrocode Suite, IHTR 2088, Sept. 1998.

[3] R. Whirley, B. Engelman and J.O. Hallquist, DYNA-3D: A Nonlinear, Explicit Three-Dimensional Finite Element Code for Solid Mechanics, User Manual, Lawrence Livermore National Laboratory Report UCRL-MA 107254, Nov. 1993.

[4] R. Whirley, B. Engelman and J.O. Hallquist, DYNA-2D: A Nonlinear, Explicit Two-Dimensional Finite Element Code for Solid Mechanics, User Manual, Lawrence Livermore National Laboratory Report UCRL-MA 110630, Apr. 1992.

[5] B. Fiessler, Private Communication, IABG Corporation, Munich, Germany, Nov. 9, 1998.

[6] R.H. Cole, Underwater Explosions, Princeton University Press, 1948.

[7] P. Chambers, H. Sandusky, F. Zerilli, K. Rye and R. Tussing, Pressure Measurements on a Deforming Surface in Response to an Underwater Explosion, CP429, in: Shock Compression of Condensed Matter, Schmidt, Dandekar and Forbes, eds.,
The American Institute of Physics, 1998.

[8] H. Sandusky, P. Chambers, F. Zerilli, L. Fabini and W. Gottwald, Dynamic Measurements of Plastic Deformation in a Water-Filled Aluminum Tube in Response to Detonation of Small Explosive Charge, 67th Shock and Vibration Symposium, Monterey, CA, Nov. 1996.

[9] A. Wardlaw, R. McKeown and J. Luton, Coupled Hydrocode Prediction of Underwater Explosion Damage, 48th Annual Bomb and Warheads Technical Symposium, Monterey, CA, May 11-14, 1999.

[10] A. Wardlaw, R. McKeown, J. Luton, M. Bormann, U. Andelfinger, R. Tewes and J. McKirgen, Coupled Hydrocode Prediction of Benchmark Tests, 70th Shock and Vibration Symposium, Albuquerque, NM, Nov. 1999.

[11] V. Lombardi, D. Package and C. Joseph, Verification of MSC/DYTRAN for Doubly Wetted Interface (DWI) Response to an UNDEX Event, 70th Shock and Vibration Symposium, Albuquerque, NM, Nov. 1999.

[12] E. Gutlin and G. Zimmermann, Studies Under the GermanUnited States Project Agreement: Computer Codes for Predicting Underwater Explosion Effects, Doubly Wetted Interface (DWI) Experiments (Part II), Aug. 1998. 

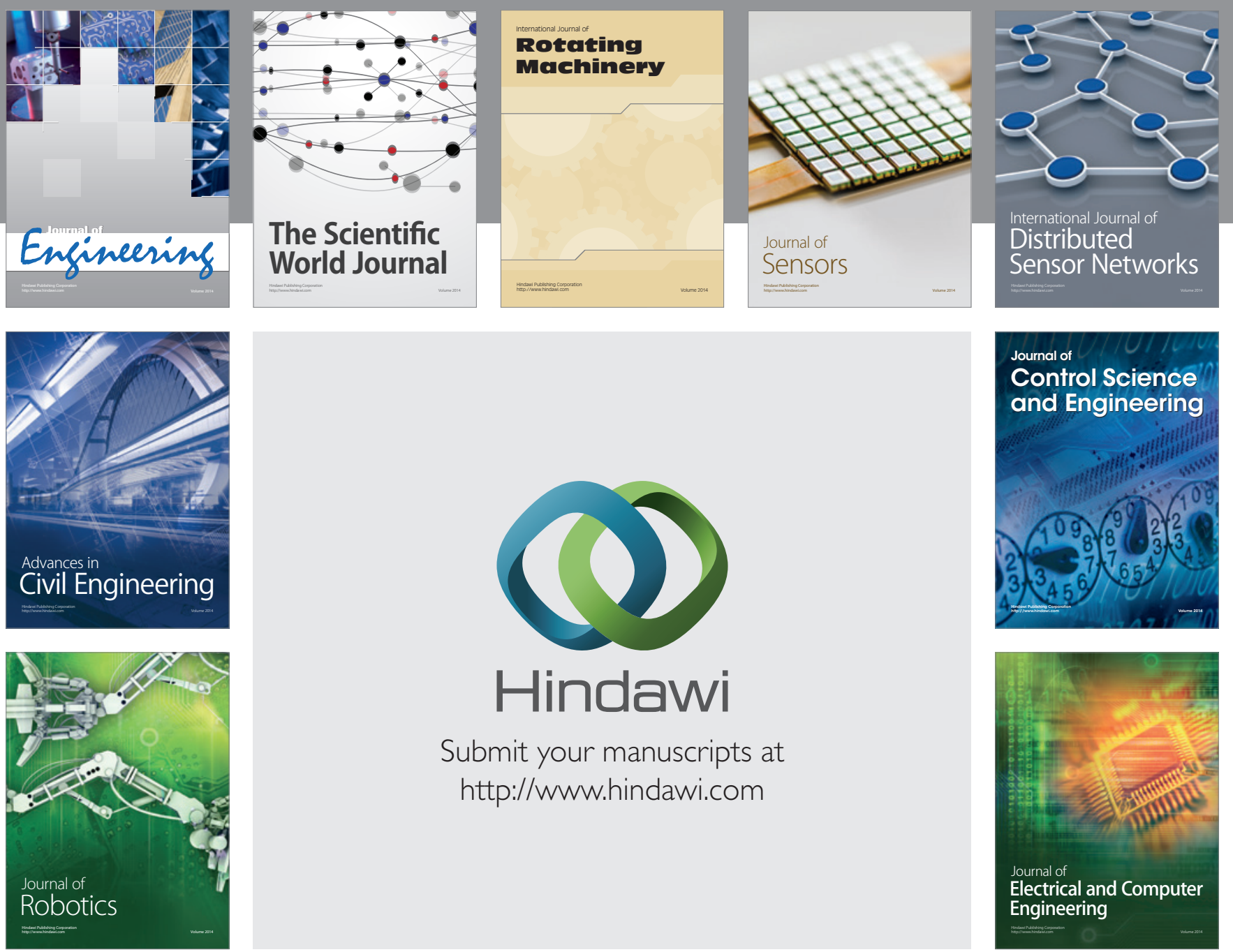

Submit your manuscripts at

http://www.hindawi.com
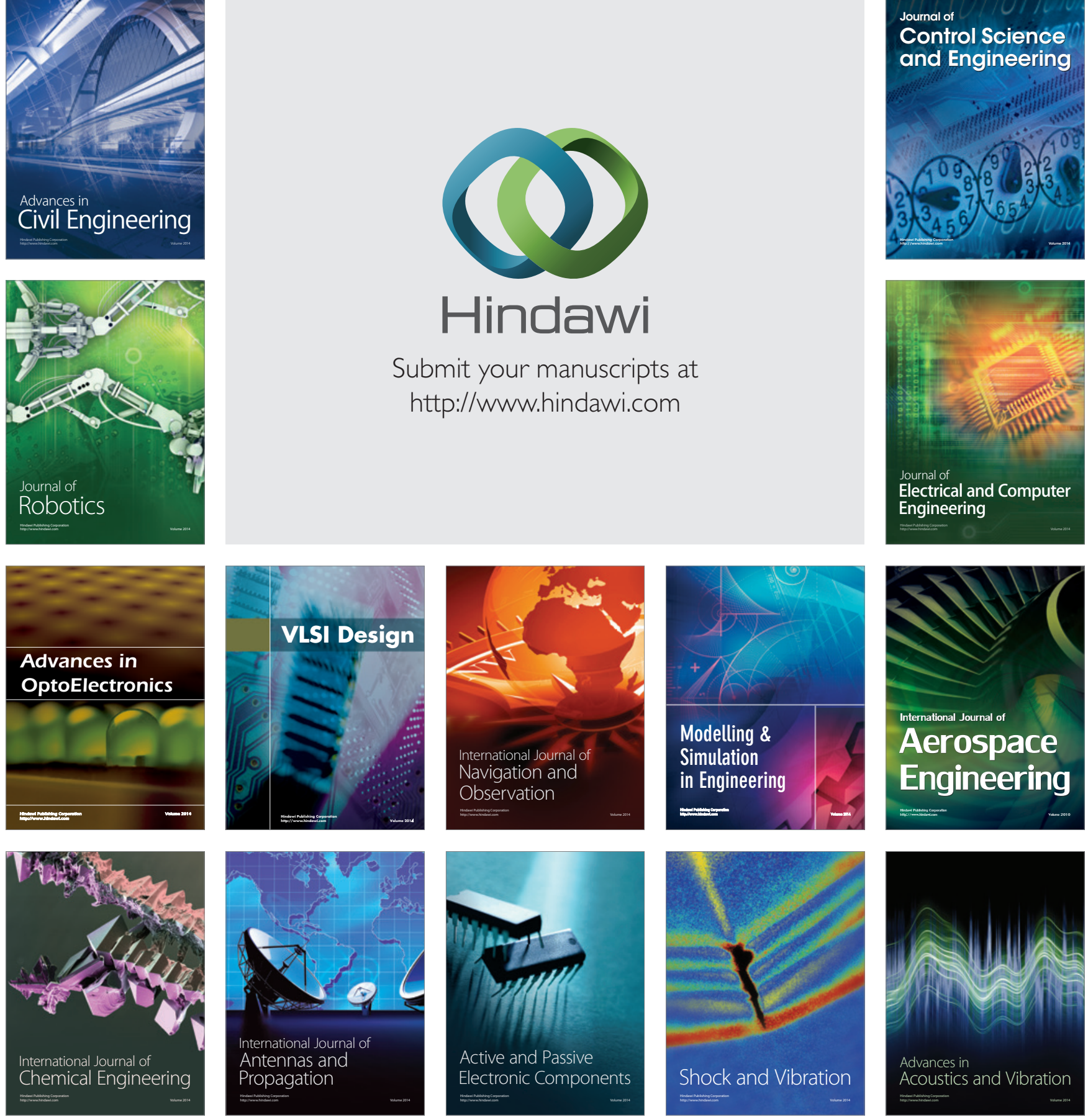